\title{
Reactivation of Tabun-inhibited Acetylcholinesterase Investigated by Two Oximes and Mutagenesis ${ }^{\dagger}$
}

\author{
Maja Katalinić and Zrinka Kovarik* \\ Institute for Medical Research and Occupational Health, P.P. 291, HR-10001 Zagreb, Croatia
}

RECEIVED DECEMBER 17, 2010; REVISED JULY 10, 2011; ACCEPTED JULY 27, 2011

\begin{abstract}
The reactivation of tabun-inhibited AChE site-directed mutants assisted by two bispyridinium oximes, K048 ( $N$-[4-(4-hydroxyiminomethylpyridinio)butyl]-4-carbamoylpyridinium dibromide) and K033 (( $N, N^{\prime}$-butano)bis(2-hydroxyiminomethylpyridinium bromide) was studied to analyse the constraints on oxime-assisted reactivation. AChE was modified within the acyl (F295L, F297I) and choline (Y337A) binding site of the active site gorge. Results show that introduced mutations affected both the affinity of phosphorylated enzyme for oximes and the rate of nucleophilic displacement of phosphoryl moiety from the catalytic serine. Mutations significantly lowered the overall reactivation efficacy of K048, but slightly enhanced the potency of K033 to reactivate tabun-inhibited AChE. It seems that the replacement of aromatic residues with the aliphatic ones at the acyl and choline binding site greatly interfered with the stabilization of the oxime's pyridinium ring(s) within the active site gorge needed to obtain the proper orientation of the oxime group toward the phosphorylated active site serine. (doi: 10.5562/cca1815)
\end{abstract}

Keywords: acetylcholinesterase, butyrylcholinesterase, nerve agents, oxime, protection, reactivation

\section{INTRODUCTION}

A high toxicity of nerve agent tabun arises from the irreversible inhibition of acetylcholinesterase (AChE; EC 3.1.1.7), important enzyme in neurotransmission. The inhibition of AChE activity results in accumulation of neurotransmitter acetylcholine at vital cholinergic sites, which in turn leads to life-threatening toxic manifestations. Currently used therapy relies on anticholinergic atropine and oximes as reactivators of tabuninhibited AChE. ${ }^{1}$ However, this approach still has its limitations mostly due to the inability of currently applied oximes (TMB-4, obidoxime, HI-6 and 2-PAM) to efficiently reactivate AChE particularly when it is phosphorylated by tabun. ${ }^{2,3}$ Therefore, solutions for improvement of the therapy are being intensively considered. ${ }^{4,5}$ One of the approaches suggests employment of enzyme scavenger capable of neutralizing tabun rapidly before it reaches targeted synaptic AChE. In this field of research, along with the endogenous butyrylcholinesterase (BChE, EC 3.1.1.8), site-directed AChE mutants could become an important tool. Moreover, the mutant enzyme in combination with oxime in a pair could act as a pseudo catalytic scavenger of organophosphates and be applied in detoxification or decontamination. ${ }^{6}$
The active site gorge of AChE is lined largely by aromatic side chains contributing to a well-defined acyl pocket and choline binding site at the base of the gorge. $^{7-9}$ Therefore, the orientation of the oxime and conjugated organophosphate within narrow confines of the gorge as well as the rates of nucleophilic attack by oxime at the conjugated phosphorus atom become important determinants of the reactivation mechanism. ${ }^{10,11}$ Moreover, a crystallographic study of tabun-inhibited mouse AChE revealed conformational changes of certain residues resulting in decreased active-site gorge. ${ }^{12}$ It become apparent that reactivation of phosphorylated AChE assisted by oximes could be optimized by altering the active site configuration through site-directed mutagenesis. ${ }^{11,13,14}$

In this paper, we investigated the reactivation of tabun-inhibited AChE site-directed mutants assisted by two bispyridinium oximes, K048 ( $N$-[4-(4-hydroxyiminomethylpyridinio)butyl]-4-carbamoyl-pyridinium dibromide) and $\mathrm{K} 033$ ((N,N'-butano)bis(2-hydroxyiminomethylpyridinium bromide). Both oximes were previously examined as reactivators of human native $\mathrm{AChE}$ and BChE inhibited by tabun. ${ }^{15,16} \mathrm{~K} 048$ was efficient reactivator of $\mathrm{AChE}$, while $\mathrm{K} 033$ was the only oxime which reactivated tabun-inhibited $\mathrm{BChE}$ to some

\footnotetext{
$\dagger$ Presented at the $10^{\text {th }}$ Congress of the Croatian Society of Biochemistry and Molecular Biology held in Opatija, Croatia, September 15-18, 2010.

* Author to whom correspondence should be addressed. (E-mail: zrinka.kovarik@imi.hr)
} 
extent. ${ }^{15,16}$ In this study, AChE was modified within the acyl pocket (F295L, F297I) and choline binding site (Y337A) of the active site gorge by replacing the aromatic amino acid residues with the aliphatic ones mimicking amino acids of $\mathrm{BChE}$ active site. By using $\mathrm{AChE}$ mutants and $\mathrm{K} 048$ and $\mathrm{K} 033$, two oximes of different molecular structure, differences in reactivation efficacy have been analyzed with the objective of enhancing reactivation rates.

\section{EXPERIMENTAL}

\section{Chemicals}

Studied oximes K048 ( $N$-[4-(4-hydroxyiminomethylpyridinio)butyl]-4-carbamoylpyridinium dibromide) and K033 ( $N, N$ '-butano)bis(2-hydroxyiminomethylpyridinium bromide), shown in Figure 1, were provided by Dr Kamil Kuča (Department of Toxicology, Faculty of Military Health Sciences, Hradec Králové, Czech Republic). Tabun [ethyl N,N-dimethylphosphoroamidocyanidate] was purchased from NC Laboratory, Spiez, Switzerland. Stock solution of oximes were prepared in water and of tabun in isopropyl alcohol. Further dilutions of these compounds were made in water before use. Cholinesterase substrate acetylthiocholine iodide (ATCh), and thiol reagent, 5,5'-dithiobis(2-nitrobenzoic acid) (DTNB), were purchased from Sigma Chemical Co., St. Louis, MO, USA. Final concentration of ATCh in experiments was $1.0 \mathrm{mmol} \mathrm{dm}^{-3}$ and of DTNB was $0.3 \mathrm{mmol} \mathrm{dm}^{-3}$.
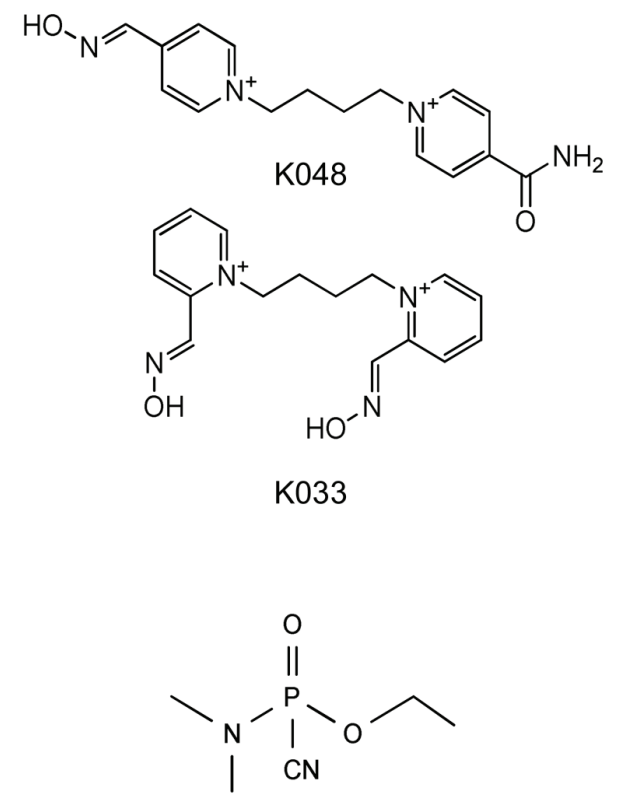

Tabun

Figure 1. Structure of tabun and tested oximes K048 and K033.

\section{Enzymes}

Recombinant wild-type and mutant mouse cholinesterases (Y337A, F295L/Y337A, F297I/Y337A) were provided by Professor Palmer Taylor (University of California at San Diego, La Jolla, CA, USA). Preparation, expression, purification and characterization of recombinant wild-type mouse $\mathrm{AChE}$ and $\mathrm{AChE}$ mutants were described in detail in previous studies. ${ }^{8,17,18}$

\section{Reactivation Experiments}

Wild-type and mutant enzymes (concentrations between $0.01-1.0 \mu \mathrm{mol} \mathrm{dm}{ }^{-3}$ ) were incubated with $5 \mu \mathrm{mol} \mathrm{dm}{ }^{-3}$ tabun until inhibition was greater than $90 \%$. Such inhibited enzyme was passed through a Sephadex G-50 spin column (Roche Diagnostic GmbH, Mannheim, Germany) to remove excess of unconjugated tabun. After filtration enzyme was incubated with oxime $\left(0.05-5.0 \mathrm{mmol} \mathrm{dm}^{-3}\right)$ in $0.1 \mathrm{~mol} \mathrm{dm}^{-3}$ sodium phosphate buffer $(\mathrm{pH}=7.4)$ containing $0.01 \% \mathrm{BSA}$ at $25{ }^{\circ} \mathrm{C}$. At specified time intervals, $10 \mu^{-3}$ aliquot was diluted 100 -fold in phosphate buffer containing DTNB and with addition of ATCh residual enzyme activity was measured by the Ellman method. ${ }^{19}$ An equivalent sample of uninhibited enzyme was passed through a parallel column, diluted to the same extent as the inhibition mixture, and control activity was measured in the presence of oxime at concentrations used for reactivation. Both activities of control and reactivation mixture were corrected for oxime-induced hydrolysis of ATCh. Kinetic parameters of reactivation, constants $k_{+2}$ (maximal first order reactivation rate constant), $K_{\mathrm{ox}}$ (phosphorylated enzyme-oxime dissociation constant) and $k_{\mathrm{r}}$ (secondorder rate constant of reactivation), were calculated from experimental data obtained in at least three experiments by equations described elsewhere. ${ }^{20}$

\section{RESULTS AND DISCUSSION}

Recombinant mouse cholinesterases phosphorylated by tabun were subjected to reactivation by two bispyridinium oximes K048 and K033. The major difference between these oximes is the number and the position of oxime(s) group(s) on the pyridinium rings. Therefore their nucleophilic attack on the phosphorylated catalytic serine should have different steric constrains. Furthermore, it was shown earlier that K033 with the oxime groups in the ortho-position was less flexible than K048 with the oxime group in the para-position on the pyridinium ring due to a higher energy barrier for rotation of the bond between pyridinium ring and linker. ${ }^{20-22}$ The flexibility was also related with their efficiency in reactivation of tabun-inhibited $\mathrm{AChE}$ because only $\mathrm{K} 048$ was able to reactivate $\mathrm{AChE}$ in a short time. ${ }^{20}$ However, K033 reactivated tabun-inhibited BChE. ${ }^{16}$ 
Table 1. Reactivation of tabun-inhibited mouse recombinant enzymes by oxime K048

\begin{tabular}{clcccccc}
\hline Enzyme & Source/Type & $\begin{array}{c}{[\mathrm{K} 048] /} \\
\mathrm{mmol} \mathrm{dm}^{-3}\end{array}$ & $\begin{array}{c}k_{+2} / \\
\mathrm{min}^{-1}\end{array}$ & $\begin{array}{c}K_{\mathrm{ox}} / \\
\mathrm{mmol} \mathrm{dm}^{-3}\end{array}$ & $\begin{array}{c}k_{\mathrm{r}} / \\
\mathrm{dm}^{-3} \mathrm{~mol}^{-1} \mathrm{~min}^{-1}\end{array}$ & $\begin{array}{c}\text { React }_{\max } / \\
\%\end{array}$ & $\begin{array}{c}\text { Time } / \\
\mathrm{h}\end{array}$ \\
\hline AChE & Mouse - w.t. & $0.5-5.0$ & $0.12 \pm 0.003$ & $0.19 \pm 0.03$ & $632 \pm 114$ & 100 & 0.5 \\
& Y337A & $0.1-3.0$ & $0.0039 \pm 0.0002$ & $0.062 \pm 0.022$ & $63 \pm 22$ & 80 & 17 \\
& F295L/Y337A & $0.1-5.0$ & $0.0025 \pm 0.0002$ & $0.11 \pm 0.05$ & $23 \pm 11$ & 40 & 8 \\
& F297I/Y337A & $0.5-3.0$ & $0.00077 \pm 0.00005$ & $0.35 \pm 0.09$ & $2.2 \pm 0.6$ & 40 & 25 \\
& Human - erythrocyte $^{(\mathrm{a})}$ & $0.05-5.0$ & $0.074 \pm 0.003$ & $0.11 \pm 0.01$ & $673 \pm 67$ & 100 & 0.5 \\
BChE & Human - plasma $^{(b)}$ & $0.5-3.0$ & $0.0022 \pm 0.0002$ & $0.63 \pm 0.22$ & $3.4 \pm 1.3$ & 70 & 18 \\
\hline
\end{tabular}

(a) Published by Čalić et al. ${ }^{15}$

(b) Published by Lucić Vrdoljak et al. ${ }^{16}$

Table 2. Reactivation of tabun-inhibited mouse recombinant enzymes by oxime K033

\begin{tabular}{clcccccc}
\hline Enzyme & Source/Type & $\begin{array}{c}{[\mathrm{K} 033] /} \\
\mathrm{mmol} \mathrm{dm}^{-3}\end{array}$ & $\begin{array}{c}k_{\mathrm{+2}} / \\
\mathrm{min}^{-1}\end{array}$ & $\begin{array}{c}K_{\mathrm{ox}} / \\
\mathrm{mmol} \mathrm{dm}^{-3}\end{array}$ & $\begin{array}{c}k_{\mathrm{r}} / \\
\mathrm{dm}^{-3} \mathrm{~mol}^{-1} \mathrm{~min}^{-1}\end{array}$ & $\begin{array}{c}\text { React }_{\max } / \\
\%\end{array}$ & $\begin{array}{c}\text { Time } / \\
\mathrm{h}\end{array}$ \\
\hline AChE & Mouse - w.t. & $0.1-1.0$ & $0.0014 \pm 0.00008$ & $0.091 \pm 0.022$ & $15 \pm 4$ & 80 & 30 \\
& Y337A & $0.1-1.0$ & $0.0036 \pm 0.0001$ & $0.065 \pm 0.011$ & $55 \pm 9$ & 95 & 17 \\
& F295L/Y337A & $0.1-1.0$ & $0.0022 \pm 0.0002$ & $0.035 \pm 0.019$ & $63 \pm 35$ & 80 & 22 \\
& F297I/Y337A & $0.1-1.0$ & $0.00044 \pm 0.00006$ & $0.093 \pm 0.054$ & $4.7 \pm 2.8$ & 40 & 22 \\
& Human - erythrocyte $^{(\mathrm{a})}$ & $0.1-1.0$ & $0.0020 \pm 0.0003$ & $0.20 \pm 0.10$ & $10 \pm 5$ & 60 & 24 \\
BChE & Human - plasma $^{(b)}$ & $0.1-3.0$ & $0.0076 \pm 0.0004$ & $0.14 \pm 0.04$ & $54 \pm 16$ & 70 & 6 \\
\hline
\end{tabular}

(a) Published by Čalić et al. ${ }^{15}$

(b) Published by Lucić Vrdoljak et al. ${ }^{16}$

Therefore, in this study we investigated how residues of the active site influence the reactivation rate. We employed site-directed mutants of AChE mimicking residues of $\mathrm{BChE}$, and reactivation reactions were run over a wide concentration range of tested oximes. Determined kinetic parameters for reactivation of tabuninhibited mouse recombinant AChE by K048 and K033 are presented in Tables 1 and 2. Figure 2 presents the time course of reactivation of tabun-inhibited recombinant mouse enzymes by oximes K048 and K033 in 0.5 mmol dm ${ }^{-3}$ concentration.

K048 assisted-reactivation of AChE w.t. was as fast as that of human $\mathrm{AChE},{ }^{15}$ with the overall reactivation rate constant, $k_{\mathrm{r}}$, of $632 \mathrm{~mol}^{-1} \mathrm{dm}^{3} \mathrm{~min}^{-1}$, reaching $100 \%$ with $1 \mathrm{mmol} \mathrm{dm}^{-3}$ concentration of the oxime within 30 min. Single-site mutant Y337A had 10 times slower reactivation rate constant $k_{\mathrm{r}}$ than $\mathrm{AChE}$ w.t. reaching the maximum of $80 \%$ of reactivation in 17 hours. However, substitution of aromatic Y337 to alanine significantly increased affinity of phosphorylated $\operatorname{AChE}\left(\right.$ i.e. $1 / K_{\mathrm{ox}}$ ) for K048. This may be explained by newly formed non-productive interactions with other aromatic residues at the choline binding site like W86. The importance of interactions with aromatic residue Y337 at the choline binding site of phosphorylated $\mathrm{AChE}$ in reactivation was also shown for oxime similar to K048 in a study by Artursson et al. ${ }^{14}$ Results presented in that paper show that substitution of Y337 with phenylalanine, another aromatic residue, did not influ-
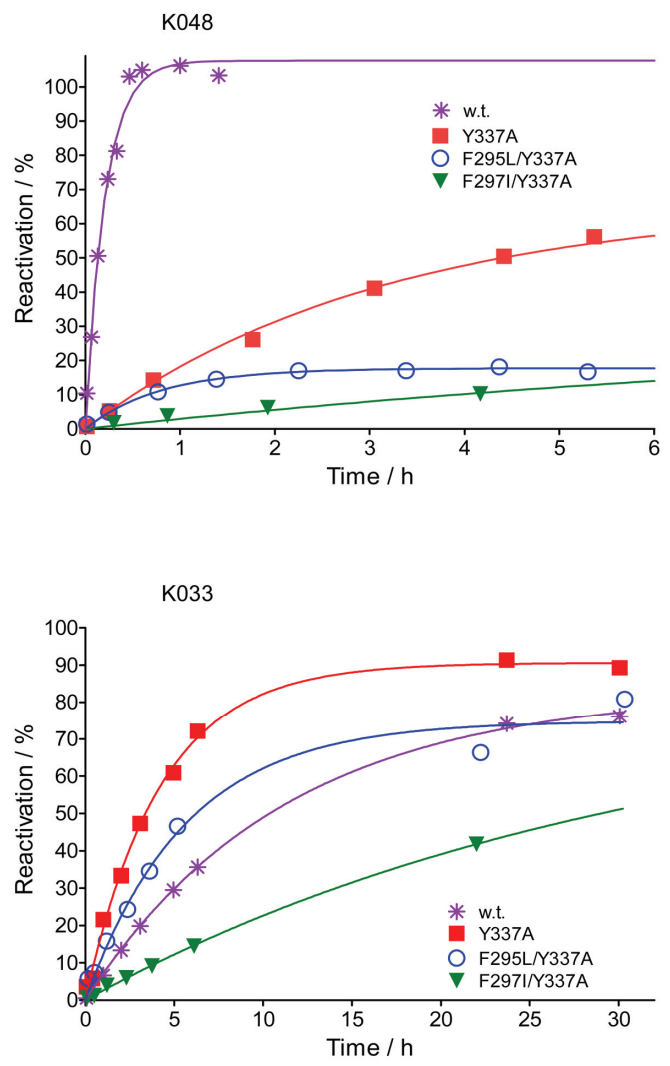

Figure 2. Time course of reactivation of tabun-inhibited recombinant mouse enzymes by oximes $\mathrm{K} 048$ and $\mathrm{K} 033$ in concentration of $0.5 \mathrm{mmol} \mathrm{dm}{ }^{-3}$. 
ence neither $k_{+2}$ nor $K_{\mathrm{ox}}$, while for Y337A mutant results were in accordance with our findings. Reactivation of double mutants, F295L/Y337A and F297I/Y337A, which carried mutations at both choline binding site and the acyl pocket, was even slower than of Y337A, reaching reactivation maximum of only $40 \%$. Since the binding affinity of these mutants was not affected compared to the AChE w.t., the major cause of losing reactivation potency here seems to be the non-productive binding of K048 with the rate of nucleophilic displacement 50 and 150 times slower than of AChE w.t., respectively. Such result indicated that the route of K048 oxime group attack comes from the acyl pocket direction and the substitution of aromatic residues in acyl pocket disturbed interactions with the K048 pyridinium ring in such a way that the transition state could not be reached.

The reactivation of tabun-inhibited AChE w.t. assisted by the ortho-oxime K033 was slow reaching $80 \%$ after $30 \mathrm{~h}$, and it is quite similar to the effect seen for human AChE earlier. ${ }^{15,16}$ Namely, the orthopositioned oxime group is not in orientation suitable for nucleophilic attack on phosphorylated catalytic serine, and the reactivation of the $\mathrm{AChE}$ is not as efficient as for para-oxime K048. ${ }^{15,16}$ For example, HI-6 as a classical ortho-oxime shows almost no efficacy for reactivation of tabun-inhibited AChE (10\% in $24 \mathrm{~h}){ }^{3,15}$ It is known from the previous study that the length of the linker between the bispyridinium rings plays an important role and that if the oxime group is orthopositioned, efficient reactivator of tabun-inhibited AChE needs to be at least heptylene-linked. ${ }^{23}$ However, for ortho-oximes both $\mathrm{AChE}$ and $\mathrm{BChE}$ have higher binding affinity than for para-oximes. ${ }^{3,16,24}$ Interestingly, in the case of $\mathrm{K} 033$, the mutagenesis resulted with a slight enhancement of the overall reactivation rates over AChE w.t. especially for mutants Y337A and F295L/Y337A. Since the affinity (i.e. $1 / K_{\text {ox }}$ ) was almost the same, the mutations seemed to enable positioning the pyridinium ring with the ortho-positioned oxime group in more appropriate orientation for the nucleophilic attack resulting with an increase of the $k_{+2}$. The strongest influence on decreasing the reactivation rate was determined for F297I/Y337A, as it was for K048, stressing again the importance of aromatic residues of the acyl pocket in reactivation of phosphorylated AChE.

Although tested AChE mutants did not show a great increase in overall reactivation by $\mathrm{K} 048$ and $\mathrm{K} 033$, they proved the relevance of aromatic amino acid configuration of the AChE active site gorge in reactivation. For para-oxime K048, mutations caused a stronger disruption of interactions and stabilization of one of the pyridinium rings during reactivation compared to the ortho-oxime K033. The enhancement achieved by $\mathrm{K} 033$ is probably too low to be of any relevance for the development of a catalytic scavenger optimized for tabun. Therefore, further screenings are necessary to identify such mutant-oxime pairs and also to gain more insight into the oxime-assisted reactivation mechanism.

Acknowledgements. This research was supported by the Ministry of Science, Education and Sports of the Republic of Croatia (Grant No. 022-0222148-2889).

\section{REFERENCES}

1. R. M. Dawson, J. Appl. Toxicol. 14 (1994) 317-331.

2. M. P. Stojiljković and M. Jokanović, Arh. Hig. Rada Toksikol. 57 (2006) 435-443.

3. F. Worek, H. Thiermann, L. Szinicz, and P. Eyer, Biochem. Pharmacol. 68 (2004) 2237-2248.

4. P. Taylor, Z. Kovarik, E. Reiner, and Z. Radić, Toxicology 233 (2007) 70-78.

5. D. E. Lenz, D. Yeung, J. R. Smith, R. E. Sweeney, L. A. Lumley, and D. M. Cerasoli, Toxicology 233 (2007) 31-39.

6. Z. Kovarik, Z. Radić, H. A. Berman, and P. Taylor, Toxicology 233 (2007) 79-84.

7. J. L. Sussman, M. Harel, F. Frolow, C. Oefner, A. Goldman, L. Toker, and I. Silman, Science 253 (1991) 872-879.

8. Z. Radić, N. A. Pickering, D. C. Vellom, S. Camp, and P. Taylor, Biochemistry 32 (1993) 12074-12084.

9. Y. Bourne, P. Taylor, and P. Marchot, Cell 83 (1995) 503-512.

10. L. Wong, Z. Radić, R. J. M. Brüggemann, N. Hosea, H. A. Berman, and P. Taylor, Biochemistry 36 (2000) 5750-5757.

11. Z. Kovarik, Z. Radić, H. A. Berman, V. Simeon-Rudolf, E. Reiner, and P. Taylor, Biochemistry 43 (2004) 3222-3229.

12. F. Ekström, Y.-P. Pang, M. Boman, E. Artursson, C. Akfur, and S. Börjegren, Biochem. Pharmacol. 72 (2006) 597-607.

13. Z. Kovarik, N. Ciban, Z. Radić, V. Simeon-Rudolf, and P. Taylor, Biochem. Biophys. Res. Commun. 342 (2006) 973-978.

14. E. Artursson, C. Akfur, A. Hörnberg, F. Worek, and F. Ekström, Toxicology 265 (2009) 108-114.

15. M. Čalić, A. Lucić Vrdoljak, B. Radić, D. Jelić, D. Jun, K. Kuča, and Z. Kovarik, Toxicology 219 (2006) 85-96.

16. A. Lucić Vrdoljak, M. Čalić, B. Radić, S. Berend, D. Jun, K. Kuča, and Z. Kovarik, Toxicology 228 (2006) 41-50.

17. Z. Kovarik, Z. Radić, H. A. Berman, V. Simeon-Rudolf, E. Reiner, and P. Taylor, Biochem. J. 373 (2003) 33-40.

18. Z. Kovarik, Z. Radić, and P. Taylor, Period. Biol. 106 (2004) 289-294.

19. G. L. Ellman, K. D. Courtney, V. Jr. Andres, and R. M. Featherstone, Biochem. Pharmacol. 7 (1961) 88-95.

20. Z. Kovarik, M. Čalić, A. Bosak, G. Šinko, and D. Jelić, Croat. Chem. Acta 81 (2008) 47-57.

21. G. Šinko, M. Čalić, A. Bosak, and Z. Kovarik, FEBS Lett. $\mathbf{5 8 0}$ (2006) 3167-3172.

22. Z. Kovarik, M. Čalić, G. Šinko, and A. Bosak, Arh. Hig. Rada Toksikol. 58 (2007) 201-209.

23. Y.-P. Pang, T. M. Kollmeyer, F. Hong, J. Lee, P. I. Hammond, S. P. Haugabouk, and S. Brimijoin, Chem. Biol. 10 (2003) 491-502.

24. Z. Kovarik, M. Katalinić, G. Šinko, J. Binder, O. Holas, Y.-S. Jung, L. Musilova, D. Jun, and K. Kuča, Chem.-Biol. Interact. 187 (2010) 167-171. 